\title{
Towards Stable Network Performance in Wireless Sensor Networks
}

\author{
Shan Lin, Gang Zhou ${ }^{\dagger}$, Kamin Whitehouse, Yafeng Wu, John A. Stankovic, and Tian He \\ Department of Computer Science, University of Virginia, Charlottesville, VA \\ ${ }^{\dagger}$ Department of Computer Science, College of William \& Mary, Williamsburg, VA \\ ${ }^{\ddagger}$ Department of Computer Science and Engineering, University of Minnesota, Minneapolis, MN \\ $\{$ shanlin, whitehouse, yw5s, stankovic $\} @$ cs.virginia.edu ${ }^{\dagger}\{$ gzhou $\} @$ cs.wm.edu ${ }^{\ddagger}\{$ tianhe $\} @$ cs.umn.edu
}

\begin{abstract}
Many applications in wireless sensor networks require communication performance that is both consistent and high quality. Unfortunately, performance of current network protocols can vary significantly because of various interferences and environmental changes. Current protocols estimate link quality based on the reception of probe packets over a short time period is neither efficient nor accurate enough to capture the dramatic variations of link quality. Therefore, we propose a link metric called competence that characterizes links over a longer period of time. We combine competence with current short term estimations in routing algorithm designs. To further improve network performance we have designed a distributed route maintenance framework based on feedback control solutions. In real system evaluations with 48 T-Motes, our overall solution improves end-to-end packet delivery ratio over existing solutions by up to $40 \%$, while reducing energy consumption by up to $22 \%$. Importantly, our solution also achieves more stable and better transient performance than current approaches.
\end{abstract}

\section{INTRODUCTION}

Extensive studies [1][15][22][3]suggest that predictable end-to-end reliability and latency are critical for many wireless sensor network applications, such as surveillance and emergency response, to meet performance guarantees. However, these wireless sensor networks are exposed to various interferences from their environments, which causes the network performance to vary dramatically and unpredictably. Therefore, it is both important and challenging to provide good network performance consistently.

In wireless sensor networks, current network protocols estimate link quality based on recent probe packets over a short time period, for example, the widely used ETX metric [2][10][35]. These solutions can achieve high reliability as long as the estimations accurately reflect the link quality when a packet is actually transmitted. Unfortunately, short term estimations may not accurately reflect the performance in indoor environments [13][34]. In our experiments on an indoor test-bed, we have observed two types of links, which we have called stable and unstable links. While the link quality of stable links stays at certain levels constantly; the quality of unstable links often change dramatically within a few seconds or a few minutes. Current short term link estimations are not effective in differentiating these two types of links, as both may maintain good qualities over a short time period. Moreover, current short term estimations are not efficient for unstable links, as the high frequency of link measurement that they require leads to increased energy consumption and interferences. Further, such links may not be accurate and when selected for routing, they may not be discarded the moment their qualities drop dramatically. As a result, end-to-end (E2E) communication quality drops and energy consumption on retransmission increases. Furthermore, the network may experience cascading route changes: newly selected routes introduce interference to other nearby routes, triggering even more packet loss, energy consumption, and route changes. The cascading route changes can result in significant E2E quality variations and energy consumption.

To address this problem, it is essential to differentiate between stable and unstable links, and give preference to stable links. We notice that these two types of links have different qualities over a long term period, e.g. tens of minutes. Therefore, we propose a new link metric competence to characterize the long term link quality. The competence metric can help choose those good and stable links for routing, and drop those currently good, but unstable ones. However, a system using only long term estimations would react too slowly to link quality changes. To react quickly and provide stable performance, we combine competence with current short term estimations in novel routing algorithm designs, selecting links that are good in both the short and the long term.

To assist in achieving stable network performance, we also design a route maintenance framework based on competence. Our framework integrates feedback control solutions at both the link and network layers. In the link layer maintenance, nodes use per-link transmission power control and retransmission control. Under certain dynamics, they help unstable links achieve stable performances at a specified level, and stable links to become more robust. The per-link performance level requirement is injected by the network layer maintenance. The network layer maintenance uses a feedback loop along an active path to translate a given E2E performance specification into per-link requirements, in order to minimize total transmission energy consumption along the path. This loop also distributes these requirements to link layer control modules at each node.

We evaluate this system on an indoor test-bed with $48 \mathrm{~T}$ Motes and show our solution improves packet delivery ratios over existing solutions by up to $40 \%$, and reduces power consumption by up to $22 \%$. In addition, real system experiments demonstrate more stable performance with less variance and better transient performance than existing solutions.

The contributions of our work are as follows:

- We have established that long term link estimation is important to achieve stable and efficient networking in 


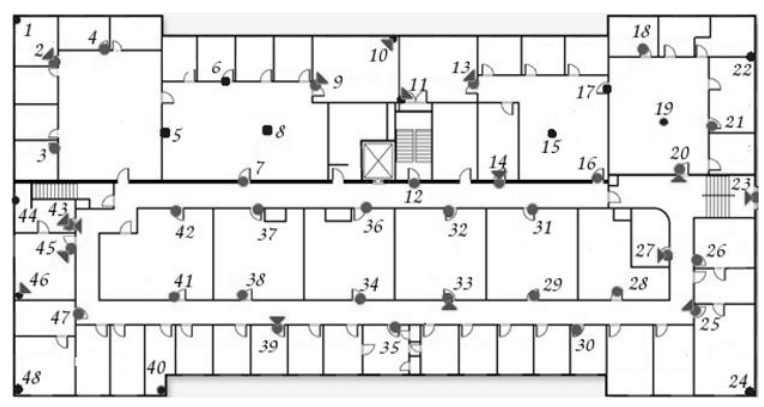

Fig. 1: The Test-bed Network Layout

the presence of interference and environmental changes. We propose the competence metric to characterize the long term quality of links for wireless sensor networks.

- We demonstrate that with a control based design, reliability of existing solutions can be further improved, and more stable and better transient performance can be achieved.

We present the competence metric design with an empirical study in Section II. In Section III, we describe the competence enhanced routing. In Section IV, we describe our feedback control framework design. In Section V, we evaluate this system on an indoor wireless test-bed. The related work is explained in Section VI, and conclusions are drawn in Section VII.

\section{The Competence Metric fOR QUALIFYING LONG TERM QUALITY}

We built a wireless test-bed in our computer science building, as shown in Figure 1. It consists of 48 T-Motes with Chipcon CC2420 low-power radios. For sensing purposes, we placed these nodes at various heights along the wall. Some of them are close to the doors, and some of them are on the top of the office cubicles.

In the first experiment, we programmed three source nodes to broadcast at a rate of 20 packets per second, while all the other nodes just listened and recorded the packets they received. We scheduled transmissions to avoid collisions. The link level retransmission and acknowledgement were disabled.

We have three observations based on these experiments:

- There are two kinds of links in the deployed system: stable links and unstable links. An example of such links is shown in Figure 2.

- There are three main causes for the link quality variations of unstable links. We distinguished these causes in the test-bed through successfully reproducing patterns of packet reception ratios (PDR) in different scenarios. These patterns are shown as small fluctuation (Figure 3 (a)), large disturbance (Figure 3 (b)), and continuous large fluctuation (Figure 3 (c)). The small fluctuations, Figures 3 (a), are mainly caused by multi-path fading of wireless signals. The large disturbances in Figure 3 (b) are caused by shadowing effect of humans, doors, and other objects. The continuous large fluctuations in Figure 3 (c) are caused by Wi-Fi interference. The variance in link qualities is largely due to a combination of instances of these three patterns.

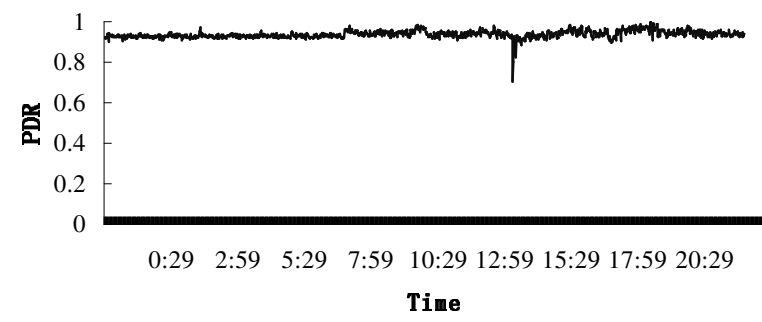

(a) A Stable Link

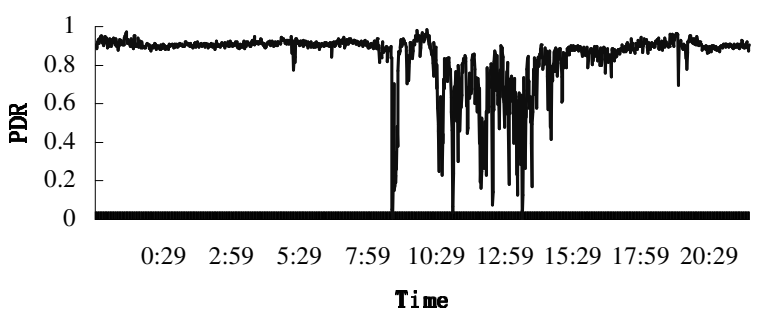

(b) An Unstable Link

Fig. 2: Stable Link vs. Unstable Link

- We also identify that there are temporal and spatial impacts of human-related activities on link quality. The quality of links in an office decreased in the morning when people walked in and started using Wi-Fi. The quality of links in the lounge demonstrated a noticeable variation at noon when people had lunch. Moreover, very similar trends were observed from links situated near each other, because human-related activities have impact on these link qualities at the same time. However, the degree of impact is different, depended on many factors, such as the distance to the interference source. Similar results were observed in indoor [13][34] and outdoor environments [15][31].

These experiments motivated us to study the stability and transient performance for wireless sensor networks in the presence of significant and rapid (sometimes within a matter of seconds) changes in communication quality. Stability and transient performance are two of the main foci of control theory, so we review the metrics in control theory before presenting our design.

Figure 4 presents the basic metrics for studying transient performance in control theory. When a certain change occurs in the system, the controlled variable deviates from the reference value. The reference value defines the level at which the controlled variable is expected to stabilize. The system is in a "steady state" when the controlled variable lies within the range between reference \pm steady state error. Otherwise, the system is in a "transient state". Another important metric is "settling time", which defines the amount of time the system takes to stabilize to a steady state when disturbances occur. The values of reference, steady state error, and settling time are specified as the control goals of the system.

These concepts and metrics are foundations of stability and transient performance analysis. However, directly applying these metrics are not reasonable because the distributed wireless network system is open and involves many uncertainties. For example, classic control systems adjust the control variable to converge to a single reference value, within the bounds 


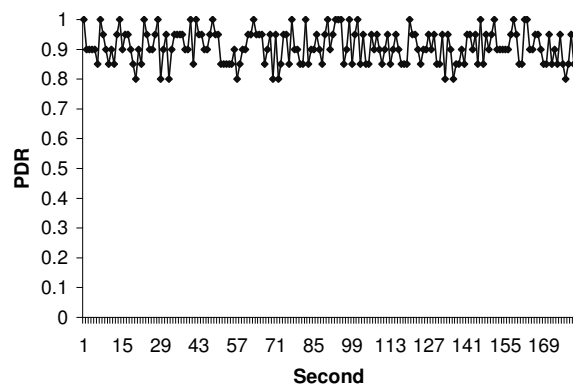

(a) Night: Radio Signal Fading

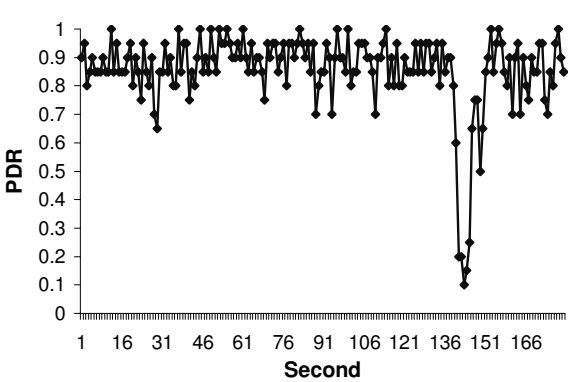

(b) Day: People Walking Around

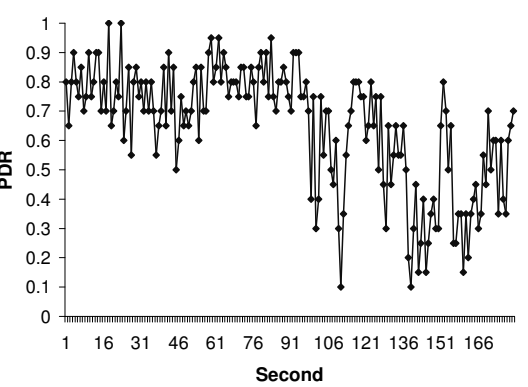

(c) Day: People Using Wi-Fi

Fig. 3: Link Quality Variation under Different Interference

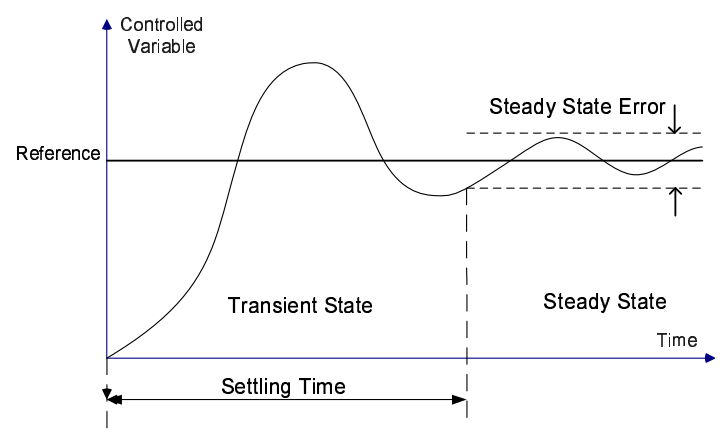

Fig. 4: Transient Performance Metric in Control Theory

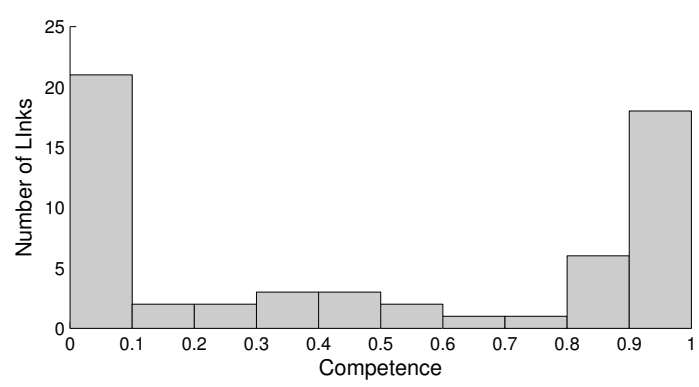

Fig. 5: Link Distribution on Link Competence defined by a very small steady state error, e.g. $2 \%$ of reference value is a reasonable design for a closed, well-modeled control system. For wireless communication quality, however, a single reference value with a very small steady range is not feasible, because fading of wireless signal can cause the packet reception ratio to vary more than $20 \%$ (Figures 3 (a)). Therefore, we need a different way to quantify stability in wireless sensor networks.

We formally define a performance metric competence. Competence is a long-term performance metric, which is based on a short term performance measure $s . s$ is a binary function indicating whether the current signal is within a desired range. The metric $c(t)$ for competence value at time $\mathrm{t}$ is defined in Equation 1.

$$
\begin{gathered}
c(t)=\alpha \cdot c(t-1)+(1-\alpha) \cdot s, \quad 1>\alpha>0 \\
s=\left\{\begin{array}{ccc}
1 & \text { if } y(t) \in\left[T_{\text {lower }}, T_{\text {upper }}\right] \\
0 & \text { otherwise }
\end{array}\right.
\end{gathered}
$$

We define $T_{\text {upper }}$ and $T_{\text {lower }}$ as the upper and lower bounds that specify a desired range for a network performance measure, such as communication quality. An exponential weighted moving average (EWMA) filter is used on the binary function $s$ that indicates whether the current communication quality is within the specified range or not. $\alpha$ is a smoothing factor indicating the weight of history when calculating the current value. $y(t)$ is the currently observed communication quality, like packet delivery ratio. The value of $c(t)$ is between 0 and 1 . If the communication quality always falls into a specified range, the value of $c(t)$ is always 1 .

There is a number of research works on link quality estimations, using filter design [19] and other indications [10]. These works provide valuable results for network protocol designs. However, as the communication quality may vary significantly within seconds, it is not effective or energy-efficient to use more probes for a more accurate estimation. The competence metric is new in focusing on characterizing long term stability of the communication quality at a desired level. It is a complementary technique to previous link estimations. Given the different spatial and temporal patterns of different links, it is beneficial to use the long term characterization of communication qualities. The long term characterization is represented by a large smoothing factor, e.g. $\alpha=0.9$, in the EWMA filter. We note that EWMA is just one of various mathematical techniques [25] to emphasize long term quality. On the other hand, because wireless communication quality can be highly variable, competence uses two bounds $\left[T_{\text {lower }}, T_{\text {upper }}\right]$ to specify a desired performance level, allowing small variation of the signal between specified bounds. In other words, this range eliminates any insignificant changes of quality.

The distribution of links from our first experiment on the competence metric is plotted in Figure 5, with a specified link quality range $[80 \%, 100 \%]$ and $\alpha=0.9 .30 .5 \%$ of the links are competent (competence $\geq 0.8$ ) and $69.5 \%$ of the links are not. These competent links have stable qualities within the specified bounds.

We also employ settling time, another important metric adopted from classical control, to quantify transient performance for wireless sensor networks. Settling time represents the amount of time a performance measure takes to deviate from and then return to a desired performance level. It quantifies a system's capacity to react to changes and return to normal performance level in the time dimension, especially when feedback control designs are applied. We use reliability as an example to demonstrate how settling time $s t$ is calculated in Equation 2. 


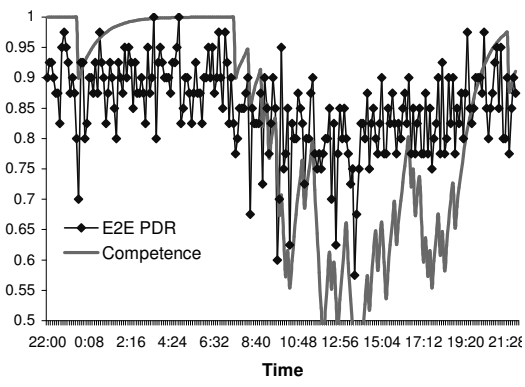

(a) E2E PDR over 24 Hours

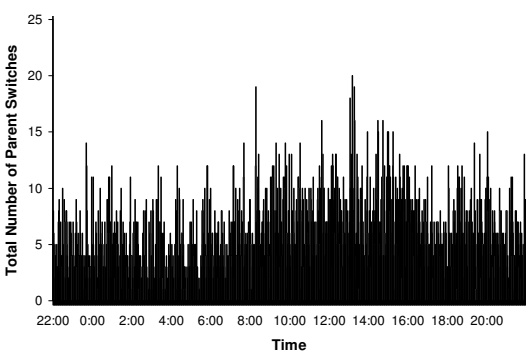

(b) Total Number of Parent Switches

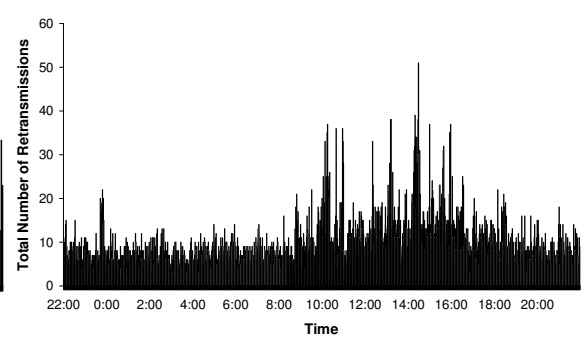

(c) Total Number of Retransmissions

Fig. 6: Performance Evaluation of MultihopLQI over 24 Hours

$$
\begin{aligned}
& s t=t_{2}-t_{1}, t_{2}>t_{1} \\
& y\left(t_{1}\right), y\left(t_{2}\right) \in\left[T_{\text {lower }}, T_{\text {upper }}\right] \\
& \wedge \forall t \in\left[t_{1}, t_{2}\right], y(t) \notin\left[T_{\text {lower }}, T_{\text {upper }}\right]
\end{aligned}
$$

The $\beta$ factor [34] is a recent metric to quantify the correlations among successes and failures of transmissions at packet level. Different from the $\beta$ factor, settling time focuses on performance resilience at a desired level with unexpected disturbances.

In order to explain the importance of stability and transient performance, we consider the example of VigilNet [14], a military surveillance system deployed on battlefields. In this application, data packets are required to be delivered to a base station with a bounded reliability, say [80\%, 100\%]. This range is much bigger than the range defined by steady state error in classic control theory, and this range is required by the application. The lower PDR bound is chosen for guaranteeing a specified surveillance quality. For tracking mobile targets, data packets must be delivered above a certain rate. If the PDR is less than a lower bound, say $80 \%$, important traces of the target may be missing. Moreover, missing important traces may lead to the inability to distinguish two targets moving closely together. Though the upper PDR bound can be set as $100 \%$, most of multi-hop communication paths in this application are set lower for the sake of energy efficiency. Achieving perfect quality consumes significantly more energy than a reasonable communication quality $(95 \%)$, due to significantly increased control overhead. In this scenario, a reasonably good communication quality meets the application goals. Similar idea applies for a number of environmental data collection applications [11] [31]. Generally, perfect communication quality is unnecessary for these applications, so long as constant good performance quality is achieved. An acceptable settling time is also required for VigilNet to successfully capture the traces of a target, in case the system performance is compromised or disturbed. If the settling time is too long, a high speed target on the edge of the surveillance area may pass across without being detected. In other applications, the settling time is also an important measure of how consistently the system can perform under significant changes.

We conducted another set of experiments to study the performance of existing protocol MultihopLQI [26] under the presence of unstable links. We ran the default configuration of MultihopLQI on our indoor test-bed for 24 hours. There were 8 source nodes, each generating 1 packet per 10 seconds. These packets were sent to a base station via multi-hop paths.
As suggested by previous studies [27] [20], this traffic load should not cause packet loss due to queue overflow.

We make three observations from this experiment:

- The end-to-end PDR varies significantly, especially during the daytime, as shown in Figure 6 (a). The plotted data represents the E2E PDR from a source to the sink. In this example, we observed that E2E PDR is around 90/

- The total number of parent switches increases significantly during the day, as shown in Figure 6 (b). This result implies that nodes do not stick to the good and stable links, but often choose unstable links. When the qualities of unstable links drop, the number of parent switches increases. Furthermore, cascading route changes occur: new routes can cause interference with other nearby routes, triggering even more packet losses, energy consumption, and route changes. Such route changes do not improve but degrade the end-to-end PDR. These cascading route changes should be avoided in network protocol design for highly dynamic networks. Figure 6 (c) presents the total number of retransmissions. As a result of selecting unstable links and cascading route changes, the number of retransmissions required during daytime increases substantially, which consumes more energy.

- There were 27 times that this E2E PDR deviated from the desired range. For example, a drop of E2E PDR occurs between $1 \mathrm{pm}$ and $2 \mathrm{pm}$ which lasts for about 60 minutes. Overall, the stability and transient performance of current protocol is not satisfactory.

\section{Competence Enhanced Routing}

In this section, we explain how we adapted the distance vector algorithm to exploit the competence metric for improved route performance in highly dynamic environments. First, we review the distance vector algorithm. The distance vector algorithm is based on a cost function, describing the resource needed to perform an operation, such as energy. Let us denote the cost of a link from node $i$ to node $j$ as $A_{i j}$, and the cost of the minimum cost route from node $i$ to the destination as $B_{i}$. The distance vector algorithm can efficiently calculate $B_{i}$ for all nodes by first having all nodes $i$ calculate $A_{i j}$ for all neighbors $j$. Then, each node chooses its parent node to be the neighbor $k$ that minimizes that value $B_{i}=A_{i k}+B_{k}$. The distance vector algorithm can be performed in a distributed fashion by having each node $i$ broadcast its own estimate of 
$B_{i}$ every time it changes. The algorithm starts when the sink node broadcasts the value $B_{\text {sink }}=0$. All neighbors of the sink estimate their own values of $B_{i}$, and the process repeats until the values at all nodes converge. If we define $A_{i j}$ to be the ETX on the link from $i$ to $j$, then $B_{i}$ is the cost of the route from node $i$ to the base station with the smallest number of expected transmissions.

The distance vector algorithm can easily be adapted to exploit the competence metric. We propose two solutions: 1) a node can choose its parent $k$ as the node with the most competent link from among all nodes $j$ with low values $B_{j}$; or 2 ) a node can choose its parent $k$ as the node with lowest value of $B_{j}$ from among all nodes $j$ to which it has a competent link. The choice between these schemes depends on application requirements, as well as the quality of links and the dynamics of the environment.

In scheme 1 , a node periodically selects a neighbor $k$ to be its parent node. This neighbor is selected via two steps. First, the node selects the lowest value $\hat{B}$ from among the values of all neighbor nodes. It then selects the set of all neighbors $j$ with values close to the lowest value: $j: B_{j} \leq R \cdot \hat{B}$, where $R$ is a specified range parameter such as $120 \%$. Second, the node selects the neighbor with the highest competence value among this set of neighbors as the forwarding node.

In scheme 2, a node first periodically selects the highest competence value of all its neighbor nodes. It then selects the set of all neighbors $j$ with competence values close to the highest one: $j$ : Competence $_{j} \geq T$. Competence lowest $_{\text {, where }}$ $T$ is a specified range parameter, such as $80 \%$. Subsequently, the node selects its parent to be the neighbor with the lowest value $B_{j}$ from among all nodes $j$ in this set.

Routing scheme 1 uses the competence metric to break ties between routes that are otherwise equivalent in terms of cost and performance. The definition of a tie is defined by the parameter $R$. This algorithm allows the routing scheme to tolerate small variations as specified by the competence bounds, but reacts to big variations via parent switches. This scheme should be used when performance is more important than robustness to network dynamics. This would likely be true in networks where network dynamics affect the routing algorithm, but do not overwhelm it.

Routing scheme 2 chooses routes that are the least susceptible to network dynamics, and breaks ties using the cost metric ETX. The definition of a tie is defined by the parameter $T$, and can be used to find cheap routes as long as they have competence levels similar to the best route. This scheme should be used when competence is more important than routing cost. Such would be the case in highly dynamic networks, or when guaranteeing performance at all times is more important than maximizing performance.

There are other factors that may influence the performance gain of our routing design, such as the network density. In a sparse network, nodes may not have many competent links available to choose from among the low cost links, but even in the worst case, the performance will be similar to the original algorithm without competence consideration.

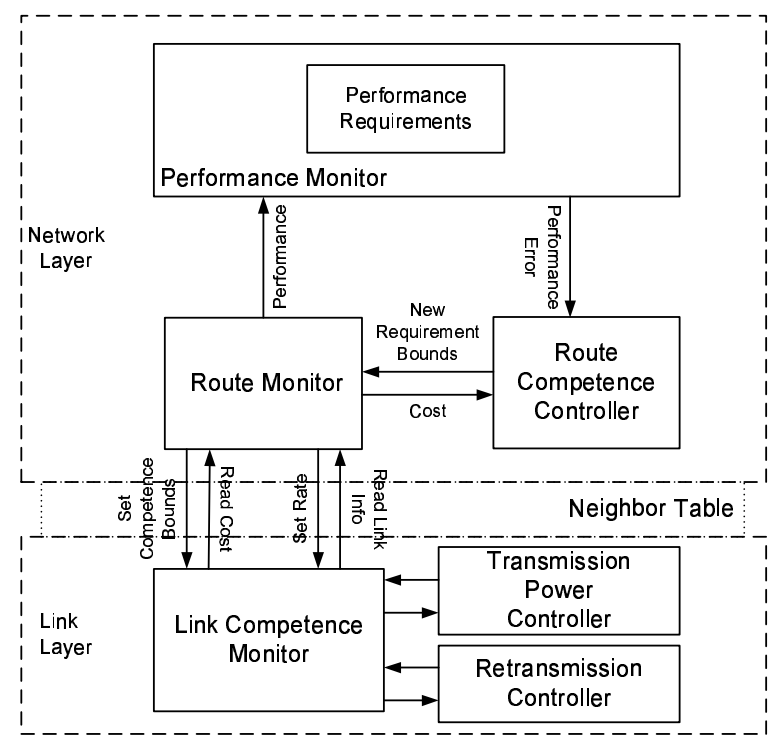

Fig. 7: Control Architecture

\section{ROUTE MAINTENANCE FRAMEWORK}

In a sparse highly dynamic network, the number of competent links can be limited. The routing structure needs to use some other links. Efficiently making use of these links is the key to reduce unnecessary route changes and improve reliability. With link quality improvement techniques [21] [28] [4] [18] some links can become competent links or more competent than before. However, those techniques have their cost in terms of energy consumption and overhead. We found that the cost is associated with long term quality of links at a specified level. Due to the high overhead to deal with variations, maintaining a stable link within certain bounds costs less than maintaining an unstable link within the same bounds. Actually, maintaining a stable link within high bounds can costs less than maintaining an unstable link within low bounds. Therefore, we propose a route maintenance framework based on competence to maintain routes and optimize maintenance cost. Given a selected path, this framework globally assigns different performance levels to links along an active path, and locally maintains assigned performance levels. This two level maintenance design is both necessary and efficient. It is because that a single link layer solution would lead to (i) local non-optimal decisions, (ii) unbalanced cost at different links, and (iii) fluctuating end-toend performance due to uncoordinated control along a path.

In competence enhanced routing, we use values of the competence metric as a routing metric; while in the route maintenance framework, we use the bounds of the competence metric as parameters for end-to-end performance control. The architecture of this maintenance framework is shown in Figure 7. The control modules are located at two layers: the network layer and the link layer. At the network layer, there are a performance monitor with specified requirements, a competence controller and a route monitor. At the link layer, there are a link monitor and controllers. We focus on reliability as the performance requirement in this work. The performance requirement consists of specified competence bounds on E2E 
PDR. Given the specified bounds, the performance monitor calculates competence based on observed E2E PDR. When competence drops below a certain threshold, E2E PDR error is passed to the route controller. With an end-to-end feedback loop along this path, the route controller collects costs from each link and allocates the stable link performance requirements in order to optimize energy consumption. Then the link performance requirements are injected to link control modules along this path. At the link layer, both the transmission power control and the retransmission control are used to enforce the link performance requirements, which are adaptive and lowcost solutions to control single link reliability.

\section{A. Link Layer Competence Maintenance}

We use power control and retransmission control as two general techniques for the link layer maintenance design. The link layer control design is shown in Figure 8. The controlled variables are the transmission power level and the number of link level retransmissions. These two controllers work independently. The link competence monitor measures PDR competence. If PDR competence drops below a certain threshold, control actions are triggered. The set points and bounds for PDR are specified by network level maintenance.

$$
\begin{gathered}
P D R(x)=1-[1-p]^{x} \\
u_{r t}(t)=u_{r t}(t-1)+\frac{\log e_{P D R}(t-1)}{\log (1-p(t-1))}
\end{gathered}
$$

The retransmission control model is based on Equation 3. The $p$ stands for the probability of successful transmission for a single attempt. We assume that the success probabilities of transmissions are independent of each other. The input $x$ is the maximum number of retransmissions. The controller form is derived from Equation 3 and shown in Equation 4. $u_{r t}(t)$ is the representation of the maximum number of retransmissions $x$. This controller takes PDR error $e_{P D R}(t)$ as input, and adjusts the number of retransmissions $u_{r t}(t)$ as output.

$$
R S S=\beta T P+\gamma
$$

The goal of transmission power control is to achieve high $p(t)$ while saving transmission energy. A control model designed in [21] is shown in Equation 5. The RSS refers to the signal strength of the link; TP represents the transmission power level applied at the transmitter of the link; $\beta$ and $\gamma$ are link specific time-varying parameters dependent on environment.

$$
\begin{aligned}
& u_{t p}(t)=u_{t p}(t-1)+K_{p}\left[e_{s}(t)-e_{s}(t-1)\right] \\
& +K_{i} e_{s}(t)+K_{d}\left[e_{s}(t)-2 e_{s}(t-1)+e_{s}(t-2)\right]
\end{aligned}
$$

Based on this adaptive control model, we extend it using a PID control shown in Equation 6. This controller takes signal strength error $e_{s}(t)$ as input, and adjusts transmission power level $u_{t p} . K_{p}, K_{i}$, and $K_{d}$ are proportional, integral, and derivative gains of the controller. To obtain the lowest settling time and highest reliability, we tuned this PID controller on different unstable links during unstable periods in the daytime and stable periods in the nighttime. We obtained two different sets of gain values in these two periods. The integral gain tuned for the unstable periods is noticeably larger than that

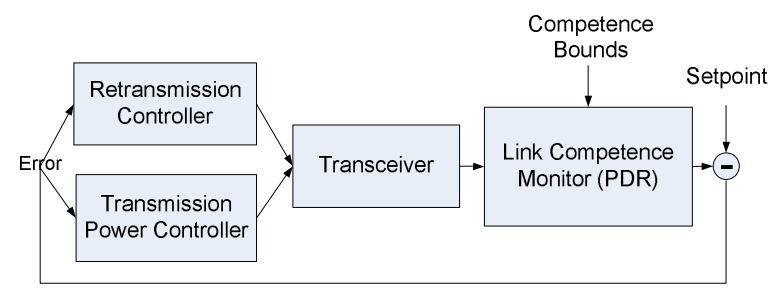

Fig. 8: Link Layer Maintenance

of the stable periods, which compensates for the quality fluctuations and optimizes settling time. The Transmission power controller uses a gain scheduling approach. The switches of gain values are triggered by the link competence monitor. When competence measure becomes lower than the setpoint, the controller starts using the gains for the unstable period. When competence measure becomes higher than the setpoint, the controller employs the gains for the stable period. We also use a conservative threshold for robust link quality estimation in the indoor environment suggested by [13] [33].

\section{B. Network Layer Competence Maintenance}

For the good and stable links, high and stable performance is maintained with a very small cost using the link layer maintenance. However, maintaining equally high performance for the unstable links is costly due to control overhead. To maintain end-to-end performance while optimizing total transmission energy consumption, our algorithm assigns competence bounds to links along an end-to-end path.

$$
\operatorname{E} 2 E P D R(k)=\prod_{i=1}^{k} P D R\left(x_{i}\right)
$$

The relation between end-to-end PDR and link PDR along a $k$-hop path is presented in Equation 7. $x_{i}$ represents the number of transmissions at hop $i$. This equation also indicates the relation between the bounds of end-to-end competence and link level competence.

$$
\operatorname{Cost}\left(x_{i}\right)=c_{t p} \cdot \sum_{i=1}^{x_{i}} i \cdot(1-p)^{(i-1)} \cdot p
$$

In Equation 8, the expected energy consumption of the link layer control at link $i$ is represented as a cost function of the transmission power level and the number of retransmissions $x_{i}$. We note that $c_{t p}$ is a constant for an attempt of transmission (including multiple retransmissions). The value of $c_{t p}$ depends on the transmission power level used.

$$
\sum_{i=1}^{k}\left(\operatorname{Cost}\left(x_{i}\right)+\text { overhead }_{i}\right)
$$

The total transmission energy consumption of an end-toend path is presented in Equation 9. There are two types of costs for each link. The $\operatorname{Cost}\left(x_{i}\right)$ is the energy consumption for transmission on link $i$ given the number of transmission $x_{i}$, as shown in Equation 8. The overhead $i$ is the energy consumption for control overhead on link $i$, such as energy consumption for feedback packets, which can be measured at each link.

Our goal is to minimize the total cost along a path while meeting the specified performance level. When the path is 


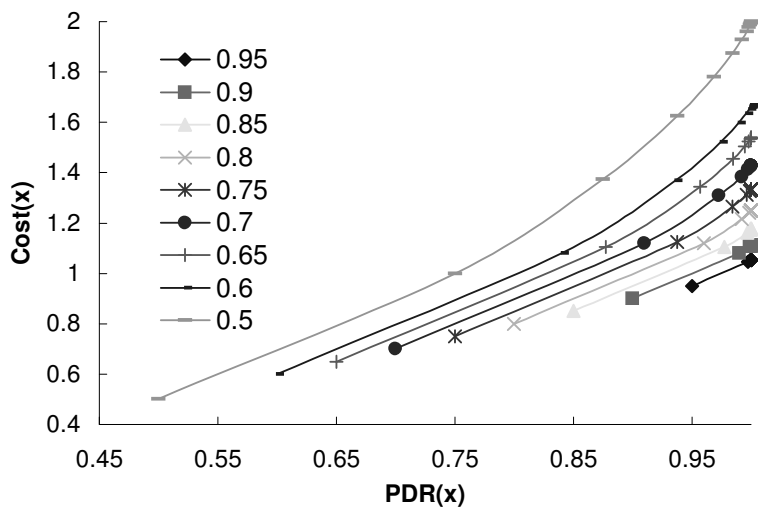

Fig. 9: Approximate Linear Relation

first established, we give every link on the path the same competence bounds. To optimize total energy consumption, high competent links should have high bounds, and low competent links should have low bounds. Mathematically, this problem is presented as follows.

$$
\begin{aligned}
& \min \sum_{i=1}^{k}\left(\operatorname{Cost}\left(x_{i}\right)+\text { overhead }_{i}\right) \\
& \text { s.t. } E 2 E P D R(k) \geq F \\
& 0 \leq p_{i} \leq 1, x_{i} \in \mathbb{N}
\end{aligned}
$$

$F$ specifies the desired end-to-end PDR. This is a nonlinear optimization problem, which can be approached by KKT conditions. We skip the construction and calculation details. However, the complexity of this problem after applying KKT is still exponential. Fortunately, we find that functions $\operatorname{PDR}(x)$ and $\operatorname{Cost}(x)$ have an approximate linear relation in their small range. We plot the relations in Figure 9. Each curve in this figure represents the relation between $P D R$ and Cost at a fixed $p$. So we can use a linear model as shown in Equation 11 to describe this relation, especially when $p$ is larger than 0.5 , which is the range of link qualities of the most useful links.

$$
\operatorname{Cost}\left(x_{i}\right)=a_{i} \cdot \operatorname{PDR}\left(x_{i}\right)+b_{i}
$$

In this linear model, $a_{i}$ and $b_{i}$ are functions of $p_{i}$. Given $p_{i}$, values of $a_{i}$ and $b_{i}$ are fixed using a least square approximation. Based on this linear model, the complexity of this optimization problem is now linear, rather than exponential. As a result, we can tell that when $a_{i} \cdot \operatorname{PDR}\left(x_{i}\right)$ are equal to each other, the total cost is minimized. The minimal cost is $\frac{\sqrt[k]{F_{i=1}^{k} a_{i}}}{k}$. And the correspondingly,

$$
P D R\left(x_{i}\right)=\frac{\sqrt[k]{F \prod_{i=1}^{k} a_{i}}}{a_{i}}
$$

Based on this Equation 12, nodes can calculate their new bounds: desired PDR. In order to do this, every node needs to know its $a_{i}$ and $\prod_{i=1}^{k} a_{i}$. $a_{i}$ is obtained from a local table storing values of $a$ and $b$, given $p$. The latter can be calculated and delivered to nodes via a feedback loop, as shown in Figure 10. In this feedback loop, a control packet is sent from source node to sink node periodically. This packet is used

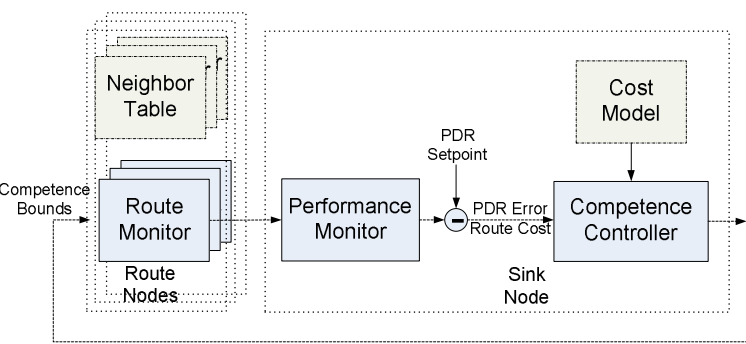

Fig. 10: E2E Feedback Loop

to calculate $\prod_{i=1}^{k} a_{i}$ hop by hop. The performance monitor at the sink node monitors the end-to-end PDR, and compares it with the specified PDR level. If there is an error, the performance monitor notifies the competence controller about the current error. The competence controller takes $\prod_{i=1}^{k} a_{i}$ and the path length as inputs and calculates $\sqrt[k]{F \prod_{i=1}^{k} a_{i}}$. This value is then sent back as feedback to every node along the path via control packets. Nodes then calculate desired PDR bounds according to Equation 12. Finally, link control maintenance can calculate the maximum number of transmissions $x_{i}$ based on Equation 12. Our control based design has limitations. For instance, control contention may affect system performance. However, the control contention rarely happens when traffic load is low, which is the case for many wireless sensor network applications. Our evaluation demonstrates that the control based approach works well in real systems. To address another potential concern, although distributed control consumes resources and introduces delay in large scale networks, in most existing wireless sensor networks, the number of nodes reporting to the same base station is less than a few hundreds. When multiple routes pass through the same link, the link and network layer's maintenance keep the parameters for every route.

\section{EVALUATION}

The most widely used link metric is the expected number of transmissions (ETX) [2][35][10][26]. Many popular data collection protocols combine the distance vector algorithm with link estimation techniques for wireless sensor networks. For example, the Mintroute [35] algorithm uses eavesdropping and an EWMA operator to estimate the probability of successful transmission over each link. It then translates these probabilities into ETX values and uses a distance vector to find routes that minimize the end-to-end ETX. The Collection Tree Protocol [9] augments Mintroute with explicit beacon messages to estimate link quality even when traffic rates are low. MultihopLQI uses the link quality indicator (LQI) defined by IEEE 802.15.4 [16] as an instantaneous link quality estimate, helping it react more quickly to changes in link quality [26]. In [10], a hybrid estimator integrates routing feedbacks and link estimates together to achieve high reliability. These protocols are currently state of the art in data collection for wireless sensor networks, and have been shown in empirical studies to have very high packet delivery ratios. 


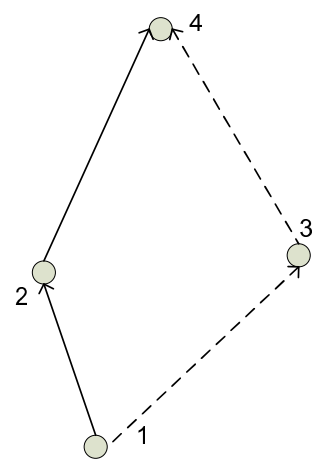

(a)

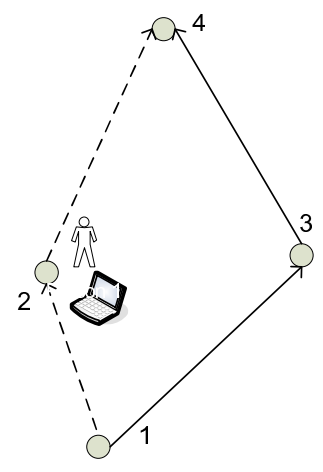

(b)
Fig. 11: Experimental Topology

Based on distance vector routing protocols, such as Multihop LQI or MintRoute [35], we have implemented a DV routing algorithm as the baseline, a competence enhanced DV routing $(\mathrm{C}-\mathrm{DV})$, and a $\mathrm{DV}$ routing (MC-DV) that is both competence enhanced and maintained. The DV routing algorithm adopts an ETX based link estimator using the EWMA filter, which is widely used in existing protocols. CDV adopts the routing scheme 1 described in Section III. In MC-DV, the end-to-end PDR bounds are set as [80\% 100\%]. The decay factor $\alpha$ for competence calculation is 0.9 . The implementation of MC-DV takes 22772B ROM and 4238B RAM.

We have conducted controlled experiments at night with 4 nodes. The topologies of these experiments are shown in Figure 11. Node 1 sends 1000 packets to base station 4 at a rate of $1 \mathrm{pkt} / \mathrm{sec}$. First we ran three algorithms when there was no interference or human activity in this area, as shown in Figure 11 (a). Then we ran another test with intentional interference near node 2, as shown in Figure 11 (b). Node 2 is hanging 4.5 feet high on the top of a cubicle and beside an office door. A student used Wi-Fi to download files in the cubicle and walked in and out using the office door from time to time.

The E2E PDRs are shown in Figure 12. From this figure we can see that in the stable periods when there is no interference or human activity, the three algorithms have almost the same PDR. The E2E PDR of path 1-2-4 is $99.3 \%$ and the E2E PDR of path 1-3-4 is $96.2 \%$. All three algorithms select the path 1-2-4 all the time, which had constant good communication quality. In this case, the use of long term estimation and maintenance do not make a difference. However, in the unstable periods three algorithms have different PDRs. This is because with interference and shadowing, the E2E PDR of path 1-2-4 was highly variable, ranging from $100 \%$ to $20 \%$. While the E2E PDR of path 1-3-4 has a little variation (around $4 \%$ ) due to weak interference (nodes 2 and 3 are located at the opposite sides of an office). DV keeps oscillating between the routing path 1-2-4 and 1-3-4, due to the short term estimation. As a result, when path 1-2-4 was selected and interference occurred near node 2, packets were lost. The routing path of C-DV converged at 1-3-4 after a few oscillations, and then the PDR settles around 94.2\%. In the MC-DV experiment, when path 1-2-4 was selected, the route maintenance increased transmission powers and number of retransmissions at nodes

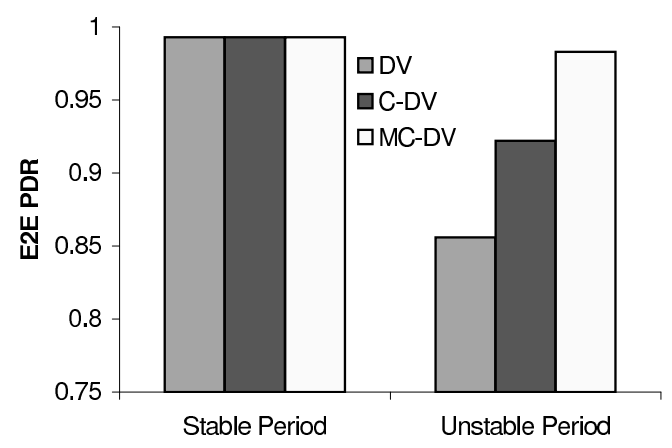

Fig. 12: Experimental Result

1 and 2. However, the PDR was still bad when interference occurred close by. Then, after MC-DV switched to path 13-4, its PDR was improved when transmission powers and retries were increased at nodes 1 and 3. And the routing path converged to 1-3-4 quickly and the E2E PDR settles at around $99 \%$. From this controlled experiment, we conclude that 1) the long term estimation used in competence helps choose stable links and improves PDR, 2) route maintenance helps improve PDR on links that are weakly interfered or shadowed, and 3) in networks with only stable links or only strongly interfered unstable links, the benefit of long term estimation and route maintenance is limited.

We also conducted nine multihop experiments in the testbed with 48 T-Motes, each experiment lasting 24 hours. We used three kinds of periodic traffic loads for communications from sources to a sink, which are typical for environmental monitoring: in traffic load 1 (L1) there were 3 source nodes, each of them sending a data packet every 20 seconds. In traffic load 2 (L2) there were 8 source nodes, each of them sending a data packet every 20 seconds. In traffic load 3 (L3) there were 8 sources, each sending a packet every 10 seconds. We note that such traffic loads do not cause message queue overflow. In this experiment, we focus on studying the performance difference between stable periods in the night and unstable periods in the day. We divided the data obtained in each 24-hour experiments into 2 parts, corresponding to a stable network period from 8PM to 8AM and an unstable network period from $8 \mathrm{AM}$ to $8 \mathrm{PM}$, and plotted them in Figure 13, Figure 14, Figure 15, and Figure 16.

In Figure 13 (a) and Figure 14 (a), we have plotted the observed average end-to-end PDR. We have also plotted corresponding standard deviations over 12 hours. We have drawn four main observations from these figures: first, DV has a much higher E2E PDR and a smaller standard deviation in the stable periods than the unstable periods, while the E2E PDRs of C-DV and MC-DV demonstrate much smaller performance differences in both stable and unstable periods. Previous evaluations have shown that ETX based routing algorithms can achieve good performances in stable networks. Our evaluations have confirmed that. For example, the E2E PDR of DV with traffic load L2 is $87.9 \%$ over 12 hours, including 8 continuous hours above $90 \%$. However, E2E PDRs of DV in the unstable periods drop significantly. This result shows that previous solutions do not work well in highly dynamic networks. Second, the differences between E2E PDRs of CDV in both stable and unstable periods and the differences 


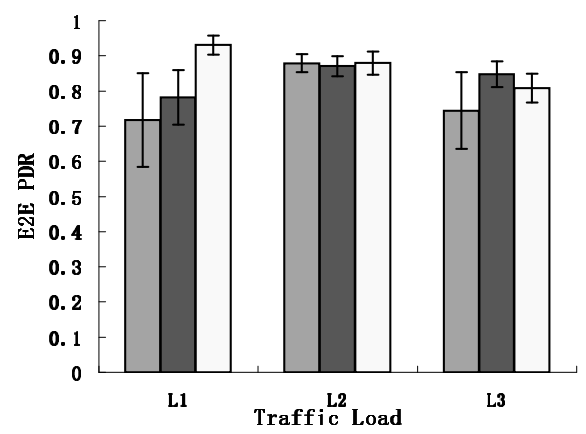

(a) Average E2E PDR

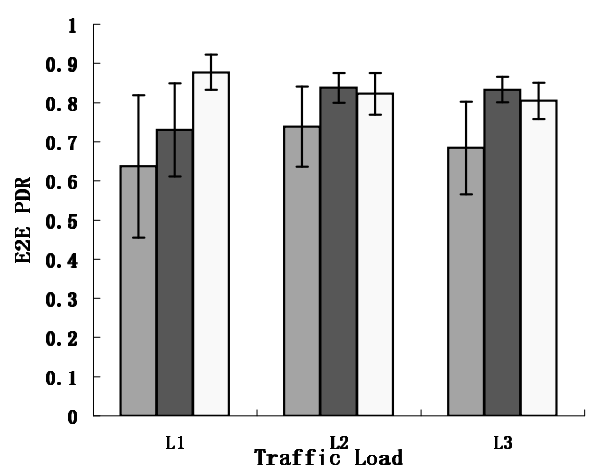

(a) Average E2E PDR

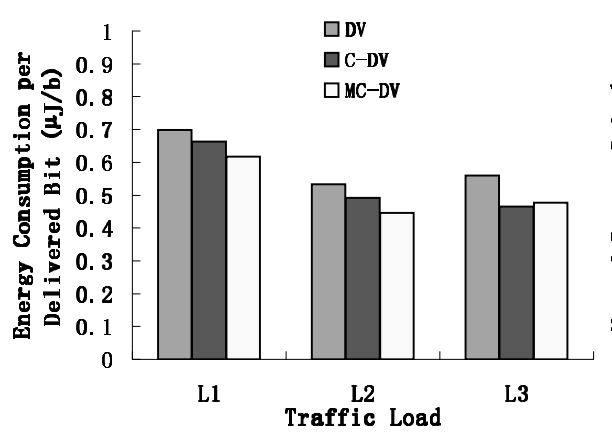

(b) Energy Efficiency

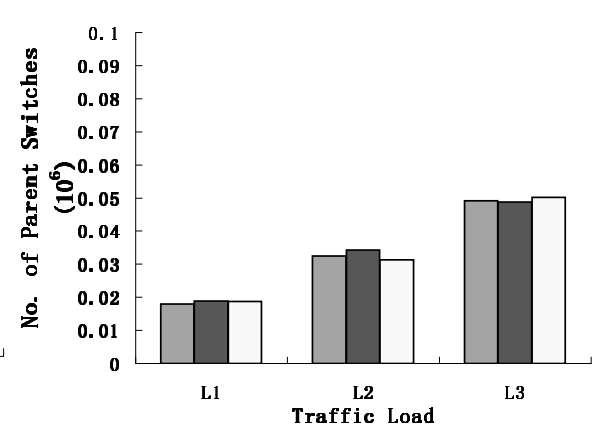

(c) Parent Switch

Fig. 13: Evaluation in Stable Periods

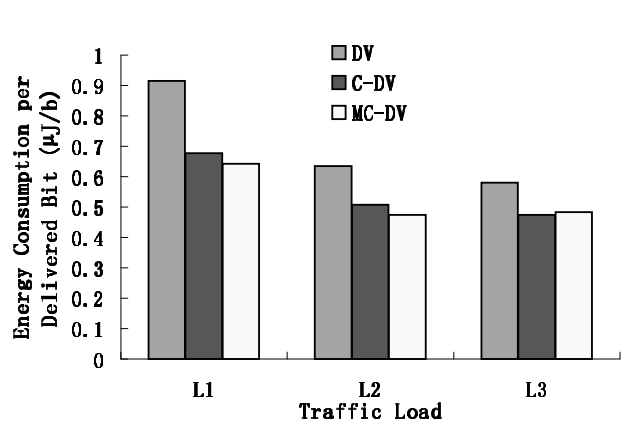

(b) Energy Efficiency

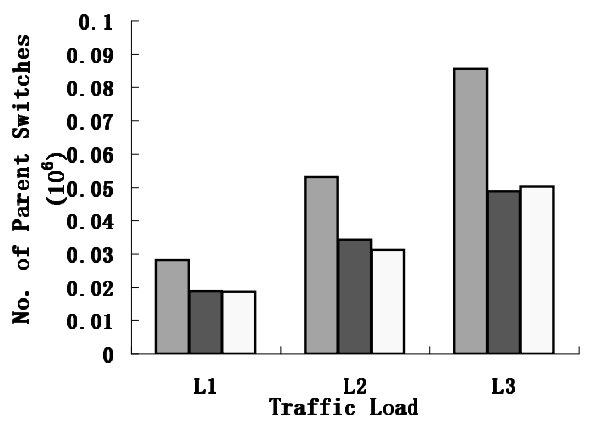

(c) Parent Switch

Fig. 14: Evaluation in Unstable Periods

between E2E PDRs of MC-DV in the same periods are less than 5\%. The standard deviations of C-DV and MC-DV are also much smaller than those of DV in all the cases. This result demonstrates that C-DV and MC-DV achieve stable and high E2E PDR in highly dynamic networks, outperforming DV. More specifically, with light traffic L1, the average endto-end PDR of C-DV is above $80 \%$ for more than $80 \%$ of the time. The average end-to-end PDR of MC-DV is above $80 \%$ for over $99 \%$ of the time. Third, MC-DV outperforms DV and C-DV with light traffic L1. This demonstrates the benefit of route maintenance for interference-free streams. Two factors that contribute to performance of MC-DV with different traffic are: interference among streams and control contention. Interference among streams affects the qualities of links. There were three streams with L1 load, while 8 streams in L2 and L3 loads. In addition, the maintenance introduces extra control packets at both link and network layers. With a heavier traffic load, the links near the base station may become unstable, while with light traffic, the interference is free and stable links are selected. On the other hand, control contentions may occur at streams near each other. For example, power controllers on two parallel links increase power alternately when their transmissions interfere, causing degrading PDR. Four, in our experiments, we found that not all observed end-to-end PDRs demonstrate obvious improvement. For other sources, we have observed smaller performance improvement than that of DV, which suggests that the improvement of competence enhanced routing depends on the density of competent links in the network. If a node has no competent links that it can use, the stability of performance will not improve much. Overall, our competence based link characterization and feedback control based stabilization are critical for achieving better network performances in dynamic wireless sensor networks.

The transmission energy efficiency is presented in Figure 13 (b) and Figure 14 (b). The transmission energy is estimated based on the total number of transmissions, the packet length, the transmission power level used for each transmission, and the control overhead. Several interesting observations can be made from this Figure: first, the energy consumption per delivered bit of all algorithms in unstable periods is higher than stable periods because that many transmissions in unstable periods are wasted without successfully delivering the packets. As shown in Figure 14 (b), in unstable periods the energy consumption per delivered bit of C-DV and MC-DV is much lower than that of DV. This result suggests that C-DV and MC-DV are more energy efficient than DV in both stable and unstable periods.

We have plotted the total number of parent switches in Figure 13 (c) and Figure 14 (c). We have two main observations: first, the numbers of parent switches of all three algorithms are similar in stable periods. This demonstrates that the characterization of stable links does not increase the number of parent switches in stable periods. Second, in the unstable periods the numbers of parent switches of DV are much higher than these of C-DV and MC-DV. This result shows that C-DV and MC-DV have successfully decreased the traffic oscillation that DV suffers in the unstable periods. We also note that as the traffic load increases, interference caused by control packets in MC-DV may increase.

We have also calculated the average competence on E2E PDR of these three algorithms in both stable periods and unstable periods, and plotted them in Figure 15 (a) and Figure 16 (a). The value of average competence represents how well 


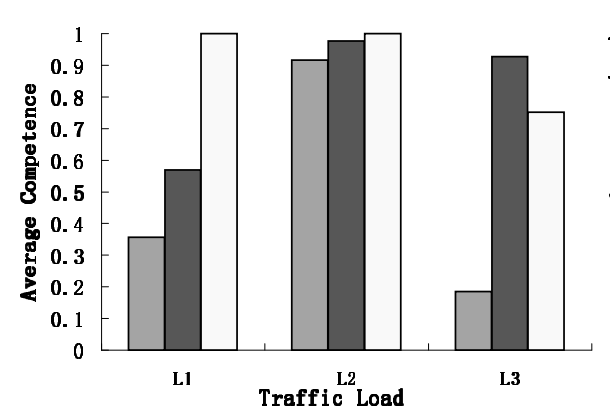

(a) Average Competence

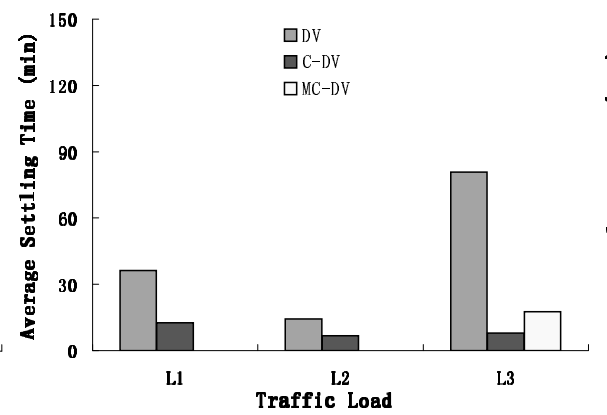

(b) Average Settling Time

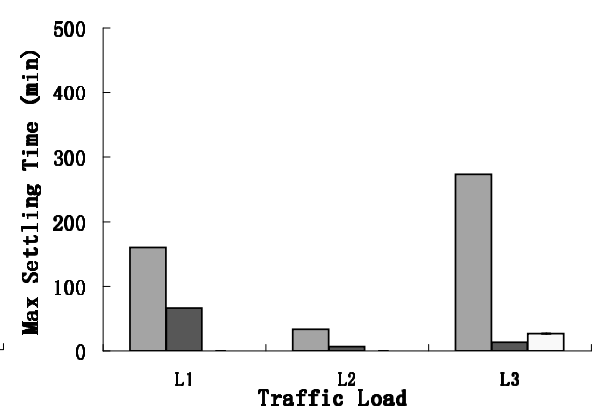

(c) Max Settling Time

Fig. 15: Evaluation in Stable Periods

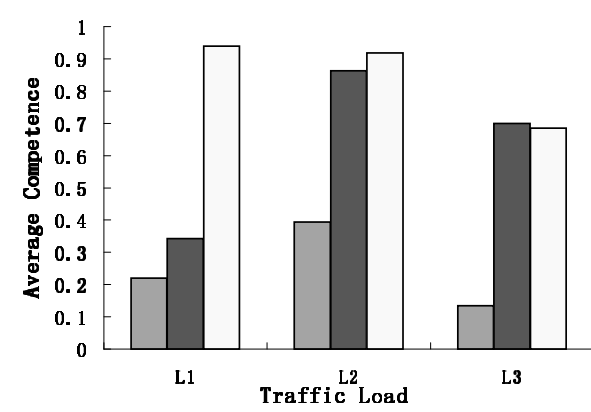

(a) Average Competence

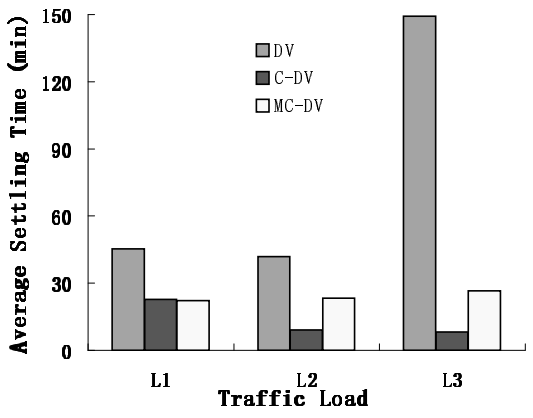

(b) Average Settling Time

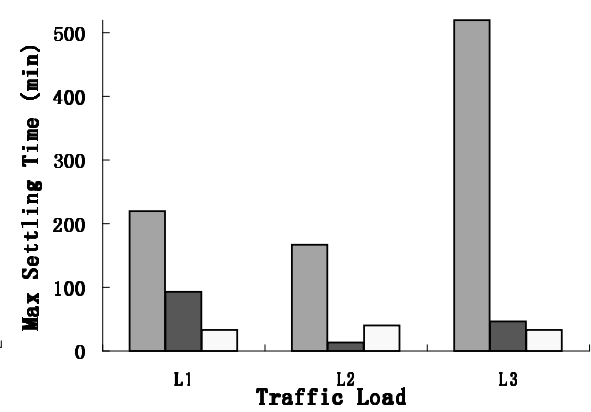

(c) Max Settling Time

Fig. 16: Evaluation in Unstable Periods

E2E PDR stays within the specified range in the long term. From these figures, we can see that average competence values of all three algorithms during stable periods are higher than corresponding ones during unstable periods. This confirms that interference and dramatic environmental changes influence the stability of E2E PDR within the specified range. On the other hand, the competence values of MC-DV and C-DV are much higher than DV during both stable periods and unstable periods. Furthermore, decreases of competence values of MCDV and C-DV during unstable periods are much less than the ones of DV. These results demonstrate that our designs achieve more stable E2E PDR than the previous design. We have calculated the average settling times and maximum settling times of these algorithms, and plotted them in Figure 15 (b) (c) and Figure 16 (b) (c). The trends of average settling times and maximum settling times are similar. From these figures, the settling times are almost twice as long during unstable periods as they were during stable periods. Both C-DV and MC-DV have a much lower average settling time than does DV in all experiments.

\section{RELATED WORK}

There are a number of wireless networking protocols that use various techniques to deal with the dynamics of wireless communication quality. At the MAC layer, short term link quality estimation [19] [35] [10] [36] [24]is critical, but we established that the long term link quality estimation is also important when link qualities can vary dramatically. We propose a metric competence to quantify long term link quality and help design routing algorithms and a framework to achieve stable E2E performance. In [19], the authors use statistical results to choose the best link estimation filter dynamically for mobile systems. In our work, competence metric focuses on the long term link estimation, which is used alongside the short term link estimation. We also use it in control designs to achieve stable network performance. Authors of [34] propose a $\beta$ factor to quantify the short term correlations among successes and failures of transmissions. Competence is different because it emphasizes the long term communication quality.

At the network layer, the existing routing protocols [12] [35] [17] [29] [8] [5] have developed mechanisms to select good links when link quality changes. In this work, we have demonstrated that selection of long term good links is also critical for high reliability and reducing cascading route changes. In our routing algorithm design, we use the competence metric in addition to previous link metrics to achieve better performance.

Many control based designs have been proposed for E2E quality of service in computing systems. These studies present elegant designs in different systems with their specific constraints, such as data servers [1], distributed real-time embedded systems [23] [32] [22], wireless sensor networks [15] [6], and Internet protocol design [30] [7]. Our control design is unique in its coordination of pairwise control at the link layer and E2E control at the network layer, based on reliability and energy constraints in wireless sensor networks.

\section{CONCLUSIONS}

This paper presents a competence metric to characterize the long term communication quality. To achieve stable performance in end-to-end communication, we incorporate the competence metric into routing protocol designs. We also propose a feedback control framework that addresses dynamics 
at both the link and network layers. Our evaluations with $48 \mathrm{~T}$ Motes have demonstrated that our design achieves satisfactory and stable network performance over time, outperforming existing protocols.

\section{ACKNOWLEDGEMENTS}

The work was supported, in part, by SAIC and NSF (CNS-0614870 and CNS-0626632). We thank the anonymous reviewers for their insightful comments. We also thank Joseph Shen and Yongjin Lu for valuable discussions.

\section{REFERENCES}

[1] T. Abdelzaher, Y. Diao, J. L. Hellerstein, C. Lu, and X. Zhu. Introduction to Control Theory And Its Application to Computing Systems. In Performance Modeling and Engineering, 2008.

[2] J. Bicket, D. Aguayo, S. Biswas, and R. Morris. Architecture and evaluation of an unplanned 802.11b mesh network. In ACM MobiCom 05 .

[3] G. Buttazzo. Research trends in real-time computing for embedded systems. SIGBED Rev., 2006.

[4] Q. Cao, T. Abdelzaher, T. He, and R. Kravets. Cluster-Based Forwarding for Reliable End-to-End Delivery in Wireless Sensor Networks. In IEEE InfoCom '07.

[5] A. Cerpa, J. L. Wong, M. Potkonjak, and D. Estrin. Temporal properties of low power wireless links: modeling and implications on multi-hop routing. In ACM MobiHoc '05.

[6] O. Chipara, Z. He, G. Xing, Q. Chen, X. Wang, C. Lu, J. Stankovic, and T. Abdelzaher. Real-time Power Aware Routing in Wireless Sensor Networks. In IWQOS' 06.

[7] D. E. Comer. Internetworking with TCP/IP Vol.1: Principles, Protocols, and Architecture. 2000.

[8] A. Dunkels, F. Österlind, and Z. He. An adaptive communication architecture for wireless sensor networks. In ACM SenSys '07.

[9] R. Fonseca, O. Gnawali, K. Jamieson, S. Kim, P. Levis, and A. Woo. Tinyos collection tree protocol. http://www.tinyos.net/tinyos2.x/doc/txt/tep123.txt.

[10] R. Fonseca, O. Gnawali, K. Jamieson, and P. Levis. Four bit wireless link estimation. In HotNets VI '07.

[11] L. Girod, M. Lukac, V. Trifa, and D. Estrin. A self-calibrating distributed acoustic sensing platform. In ACM SenSys '06.

[12] Y. Gu and T. He. Data forwarding in extremely low duty-cycle sensor networks with unreliable communication links. In ACM SenSys ' 07.

[13] G. Hackmann, O. Chipara, and C. Lu. Robust topology control for indoor wireless sensor networks. In ACM SenSys '08.

[14] T. He, S. Krishnamurthy, J. A. Stankovic, T. F. Abdelzaher, L. Luo, R. Stoleru, T. Yan, L. Gu, J. Hui, and B. Krogh. Energy-Efficient Surveillance System Using Wireless Sensor Networks. In ACM MobiSys '04.

[15] T. He, P. Vicaire, T. Yan, L. Luo, L. Gu, G. Zhou, R. Stoleru, Q. Cao, J. A. Stankovic, and T. Abdelzaher. Achieving real-time target tracking usingwireless sensor networks. In RTAS '06.
[16] IEEE 802.15.4, Wireless Medium Access Control (MAC) and Physical Layer (PHY) Specifications for Low Rate Wireless Personal Area Networks (LR-WPANs), 1999

[17] C. Intanagonwiwat, R. Govindan, D. Estrin, J. Heidemann, and F. Silva Directed diffusion for wireless sensor networking. IEEE/ACM Trans Netw., 2003.

[18] S. Katti, H. Rahul, W. Hu, D. Katabi, M. Médard, and J. Crowcroft Xors in the air: practical wireless network coding. SIGCOMM Comput. Commun. Rev., 2006.

[19] M. Kim and B. Noble. Mobile network estimation. In ACM MobiCom '01.

[20] S. Kim, R. Fonseca, P. Dutta, A. Tavakoli, D. Culler, P. Levis, S. Shenker, and I. Stoica. Flush: a reliable bulk transport protocol for multihop wireless networks. In ACM SenSys '07.

[21] S. Lin, J. Zhang, G. Zhou, L. Gu, J. A. Stankovic, and T. He. Atpc: Adaptive transmission power control for wireless sensor networks. In ACM SenSys '06.

[22] C. Lu, J. A. Stankovic, S. H. Son, and G. Tao. Feedback control realtime scheduling: Framework, modeling, and algorithms. JRTS' 02.

[23] C. Lu, X. Wang, and X. Koutsoukos. Feedback utilization control in distributed real-time systems with end-to-end tasks. IEEE Trans. Parallel Distrib. Syst., 2005.

[24] E. Miluzzo, X. Zheng, K. Fodor, and A. T. Campbell. Radio Characterization of 802.15.4 and its Impact on the Design of Mobile Sensor Networks. In EWSN '08.

[25] D. C. Montgomery. Introduction to Statistical Quality Control. John Wiley and Sons, Inc., 2005.

[26] Tinyos multihoplqi collection protocol. http://www.tinyos.net/tinyos1.x/tos/lib/MultiHopLQI.

[27] J. Paek and R. Govindan. Rcrt: Rate-controlled reliable transport for wireless sensor networks. In ACM SenSys '07.

[28] J. Polastre, J. Hill, and D. Culler. Versatile low power media access for wireless sensor networks. In ACM SenSys '04.

[29] J. Polastre, J. Hui, P. Levis, J. Zhao, D. Culler, S. Shenker, and I. Stoica. A Unifying Link Abstraction for Wireless Sensor Networks. In ACM Sensys '05.

[30] H. Schulzrinne, S. Casner, and I. H. Schulzrinne/s. Casner. Rtp: A transport protocol for real-time applications, 1993.

[31] L. Selavo, A. Wood, Q. Cao, T. Sookoor, H. Liu, A. Srinivasan, Y. Wu, W. Kang, J. Stankovic, D. Young, and J. Porter. Luster: wireless sensor network for environmental research. In ACM SenSys '07.

[32] N. Shankaran, N. Roy, D. C. Schmidt, X. D. Koutsoukos, Y. Chen, and C. Lu. Design and performance evaluation of an adaptive resource management framework for distributed real-time and embedded systems. EURASIP J. Embedded Syst., 2008.

[33] M. R. Souryal, J. Geissbuehler, L. E. Miller, and N. Moayeri. Real-time deployment of multihop relays for range extension. In ACM MobiSys '07.

[34] K. Srinivasan, M. Kazandjieva, S. Agarwal, and P. Levis. The betafactor: measuring wireless link burstiness. In ACM SenSys '08.

[35] A. Woo, T. Tong, and D. Culler. Taming the Underlying Challenges of Reliable Multihop Routing in Sensor Networks. In ACM SenSys '03.

[36] J. Zhao and R. Govindan. Understanding Packet Delivery Performance in Dense Wireless Sensor Networks. In ACM SenSys '03. 